\title{
Majority relation and median representative ordering
}

\author{
Gabrielle Demange
}

Received: 12 November 2010 / Accepted: 1 March 2011 / Published online: 29 March 2011

(C) The Author(s) 2011. This article is published with open access at SpringerLink.com

\begin{abstract}
This paper presents results on the transitivity of the majority relation and the existence of a median representative ordering. Building on the notion of intermediate preferences indexed by a median graph, the analysis extends well-known results obtained when the underlying graph is a line. In contrast with other types of restrictions such as single-peakedness, intermediate preferences allow for a clear distinction between restrictions on the set of preferences characteristics and those on the set of alternatives.
\end{abstract}

Keywords Majority rule $\cdot$ Median graph · Tree - Condorcet winner · Intermediate preferences

JEL Classification $\quad \mathrm{D} 720 \cdot \mathrm{D} 710$

\section{Introduction}

The majority rule is a prominent voting rule if any. Though, there are difficulties due to the possibility of majority cycles and the non-existence of a majority winner, as illustrated by the famous Condorcet paradox. But difficulties are unavoidable as they are bound to arise in some form with any non dictatorial rule, as shown by Arrow (1963). Not surprisingly, starting with Black (1948), a large literature tries to find conditions under which the majority rule is well-behaved. This paper provides an additional contribution to this literature. It displays families of preferences that

G. Demange thanks the editor Matt Jackson and a referee for their remarks.

G. Demange $(\varangle)$

Paris School of Economics, EHESS, Paris, France

e-mail: demange@pse.ens.fr 
guarantee the transitivity of the majority rule, meaning that the majority rule is transitive no matter what the profile of individuals' preferences in the family. Preferences are characterized by a parameter and satisfy two conditions, one on the parameter space and one on how these preferences depend on the parameter. Specifically, the parameter space is a median graph and the preferences satisfy an intermediateness assumption, terms that will be explained below. Under these conditions, not only the majority rule is transitive but also it coincides with the preference ordering associated with a 'representative' characteristic.

Following Black (1948), various restrictions on preferences over a one-dimensional set of alternatives have been introduced and shown to guarantee the existence of a Condorcet winner: single-peakedness, single-crossing, order restriction, and recently top-monotonicity by Barbera and Moreno (2009), which encompasses all of them (we refer the reader to their paper for precise definitions of these restrictions and their comparison). A one-dimensional set of alternatives is however a strong limitation. Unfortunately, extending these positive results to a multi-dimensional space turns out to be disappointing. Not only the extensions of the previous properties -say singlepeakedness- fail to guarantee the existence of a Condorcet winner but also majority cycles are pervasive. A Condorcet winner exists under very specific configurations on the profile of these preferences (Kramer 1973; Plott 1967, or Demange 1983 for a survey on these issues). In other words, restrictions on the distribution of preferences within the society are necessary. Although interesting, these restrictions are so strong that they are most likely to fail. Furthermore, they are not robust to a change in the preferences of a single individual: if the restrictions are satisfied, a single change typically leads to cycles, thereby precluding a general prediction of the majority mechanism. As a result, the strategy-proofness of the majority rule has no meaning since the majority choice is not well-defined for most of the profiles.

This paper shows that the positive aggregation results that hold on a line can be extended if the preferences' characteristics belong to a median graph. The alternative space, in particular its dimension, does not matter, under the proviso that the intermediateness assumption on preferences is satisfied.

The analysis relies on the two separate concepts of median graph on one hand and intermediate preferences on the other hand. A graph on a set defines a betweenness relationship on the elements in the set, such as the natural one if the graph is a line. The graph is median when this betweenness relationship satisfies a simple requirement: any triple has a unique median. Important examples of median graphs are trees, which include lines as a particular case, and hypercubes, composed with all points of coordinate 1 or 0 . The main property used in this paper is that any profile of characteristics in a median graph admits a local Condorcet characteristic, namely a characteristic that beats all its neighbors for the graph distance (Bandelt and Barthélémy 1984).

Intermediateness of preferences preserves the betweenness structure of the characteristics space in the following sense. The order associated to a characteristic that is between two other characteristics ranks any pair of alternatives as these two others whenever they agree on the ranking of the pair. Intermediate preferences were introduced in social choice by Kemeny and Snell (1962). Grandmont (1978) studies the majority rule for families indexed by a parameter running in (a convex set of) a multidimensional space. He shows that if a characteristic satisfies a kind of 'total' median 
property in the characteristics space, ${ }^{1}$ then the preference order associated with this characteristic coincides with the majority relation. Hence the profile can be aggregated in a consistent way and furthermore there is a 'representative' characteristic. A difficulty with this result, as argued above, is that typically a characteristics' profile admits no total median when the characteristics run in a multi-dimensional real space: some very strong conditions on the distribution of the characteristics in the profile are needed for a majority winner to exist.

Our approach builds on the same idea as Grandmont of distinguishing between the characteristics' space and the alternatives' space. Recall, the characteristics' space is a median graph and preferences are intermediate. The basic argument is as follows. First, any profile of characteristics admits a local Condorcet characteristic, which beats all its neighbors under the graph-distance. Second, under intermediate preferences, a local Condorcet characteristic results in a representative characteristic, meaning that the majority relation coincides with the ordering associated with this characteristic.

The argument makes clear the underlying structure allowing for the positive result, in particular because it disentangles properties on the structure of the preferences from those on the set of alternatives. Such kind of argument cannot be used with the single-peakedness type of condition, in which what matters in a preference ordering is the peak -the most preferred alternative- making the conditions on the dimensions of the set of preferences and that of the alternatives intertwined.

The literature on median graphs is extremely vast. Median graphs have been introduced independently in different equivalent ways in various fields, as they are related to lattice theory and important notions used in classification. Links between various definitions appear in Mulder (1980) and there are several surveys e.g. Van De Vel (1993). However, apart from some basic results for our need such as the existence of local Condorcet characteristic (Bandelt and Barthélémy 1984), this paper is almost self-contained. Most used properties are rather easy to show (once they are known), and I give their proofs.

The graph structure on the set of characteristics can be taken literally, as in the problem of locating some facilities in which individuals' characteristics are their locations on a transportation network (see e.g. Hansen and Thisse 1981, in particular for a first comparison between Condorcet winners and median points). However a graph can also represent of an ideological space, as in the hypercube with 0-1 coordinates.

Finally, median graphs have recently been used in social choice by Nehring and Puppe (2007). A betweenness relationship on the space of alternatives allows them to define single-peakedness in a general abstract framework and to analyze the possibility of strategy-proof rules. The setting is different from ours since it is the set of alternatives that is endowed with a median structure rather than the set of characteristics.

The plan is as follows. Section 2 presents the framework, introduces the basic definitions of median graph and intermediate preferences, and illustrates with some examples. Section 3 gives the main result, which states that the majority rule is cyclefree for a family of intermediate preferences on a median graph. It concludes with a comparison between intermediateness and single-peakedness.

\footnotetext{
${ }^{1}$ In a setting with an infinite number of agents, the 'total' median characteristic is such that any half hyper-plane through it cuts the characteristics profile into two sets of equal measure.
} 


\section{The framework}

The framework represents a standard situation in which a group of individuals-a society- has to choose an alternative in a feasible set and use the majority rule to do so.

There is a finite set $N=\{1, \ldots, i, \ldots, n\}$ of individuals who have preferences over these alternatives. To simplify the presentation, $n$ is assumed to be odd throughout the paper. In what follows, $X$ will denote a set of alternatives (finite or infinite), $R$ will be a preference ordering on $X$, i.e. a binary relation that is complete $(x R y$ or $y R x)$ thus reflective $(x R x)$ and is transitive. The strict relation $P$ is defined by: $x P y$ if $x R y$ but not $y R x$. The ordering $R$ is said to be strict when either $x P y$ or $y P x$ for distinct elements.

Let $R=\left(R^{1}, \ldots, R^{n}\right)$ denote the profile of the $n$ individuals' preferences relations over $X$. The majority relation $R^{m a j}$ is defined by comparing for any pair $x, y$ in $X$ the number of individuals $i$ who strictly prefer $x$ to $y, n(x, y)$, with the number of those who strictly prefer $y$ to $x, n(y, x)$. Denoting by $|A|$ the cardinality of set $A$, we have $n(x, y)=\left|\left\{i, x P^{i} y\right\}\right|$. The majority relation $R^{m a j}$ is defined by

$$
x R^{m a j} y \quad \text { if } n(x, y) \geq n(y, x) .
$$

A (weak) Condorcet winner is an alternative $x$ which is weakly preferred to any other for the majority relation:

$x$ is a Condorcet winner if $n(x, y) \geq n(y, x)$ for each $y$ in $X$.

Alternatively, since we have $y P^{m a j} x$ iff $n(y, x)>n(x, y)$, no $y$ 'beats' or dominates a Condorcet winner $x$.

We consider a family of preference orderings, denoted by $\mathcal{O}$, indexed by $\theta$ running in a set $\Theta: \mathcal{O}=\left\{R_{\theta}, \theta \in \Theta\right\}$. All individuals have preferences in $\mathcal{O}$ : $i$ 's preference ordering is $R_{\theta^{i}}$, determined by $i$ 's characteristic $\theta^{i}$. A characteristic profile is a $n$-tuple $\left(\theta^{1}, \ldots, \theta^{n}\right)$ in $\Theta^{N}$, and the set of admissible relation profiles is $\mathcal{O}^{N}$. The set $\Theta$ is assumed to be finite.

\subsection{Betweenness relationships, intermediate preferences}

There is a natural notion of betweenness for binary relationships according to which a relation is between two others if it ranks two alternatives as these two others whenever they agree on the ranking on the two alternatives (Kemeny and Snell 1962). Formally $R^{\prime}$ is said to be between $R$ and $R^{\prime \prime}$ if for all $x$ and $y$ in $X$

(a) $x R y$ and $x R^{\prime \prime} y$ imply $x R^{\prime} y$ and (b) ( $x P y$ and $\left.x R^{\prime \prime} y\right)$ or ( $x R y$ and $\left.x P^{\prime \prime} y\right)$ imply $x P^{\prime} y$.

In short the relation $R^{\prime}$ does not introduce new disagreement between $R$ and $R^{\prime \prime}$.

Consider now $\mathcal{O}$ a family of preference orders indexed by $\theta$ in a set $\Theta$. The notion of intermediate preferences assumes that $\Theta$ is endowed with a 'betweenness' relationship 
and that this relationship is inherited by the preferences. We first need to define the notion of betweenness relationship for an (abstract) set $\Theta$. There are several equivalent ways to do so and I present here the formulation in terms of graph.

Graphs and Betweenness relations A betweenness relation is derived from a graph structure that links the elements of $\Theta$. In short, a vertex is said to be between two others if it belongs to a shortest path joining them. Let us describe this formally.

A (non-directed) graph $G=(\Theta, E)$ on $\Theta$ is specified by a set $E$ of unordered pairs of distinct elements of $\Theta$. A pair $\left(\theta, \theta^{\prime}\right)$ in $G$ is called an edge between $\theta$ and $\theta^{\prime}$. In the graph terminology the elements of $\Theta$ are called vertices and two elements linked by an edge neighbors. A path between $\theta$ and $\theta^{\prime}$ is a sequence $\theta_{1}, \ldots, \theta_{j}, \ldots, \theta_{p}$ in $\Theta$ for which $\theta=\theta_{1}, \theta^{\prime}=\theta_{p}$ and the pairs $\left(\theta_{j}, \theta_{j+1}\right)$ are edges for $j \in\{1, \ldots, p-1\}$ and all distinct. In the sequel I assume that there is a path between any two pairs in $\Theta$, i.e., the graph is connected.

A shortest path linking two points is one with the minimal number of edges in a path joining the two vertices. This minimal number is defined as the distance between the two points. A vertex $\theta^{\prime}$ is between $\theta$ and $\theta^{\prime \prime}$ if it belongs to a shortest path joining $\theta$ and $\theta^{\prime \prime}$ or, equivalently, if the distance between $\theta$ and $\theta^{\prime \prime}$ is the sum of the distances between $\theta$ and $\theta^{\prime}$ and between $\theta^{\prime}$ and $\theta^{\prime \prime}$. The interval between $\theta$ and $\theta^{\prime \prime}$, denoted by $\left[\theta, \theta^{\prime \prime}\right]$, is the set of all vertices between $\theta$ and $\theta^{\prime \prime}$ :

$$
\left[\theta, \theta^{\prime \prime}\right]=\left\{\theta^{\prime}, d\left(\theta, \theta^{\prime \prime}\right)=d\left(\theta, \theta^{\prime}\right)+d\left(\theta^{\prime}, \theta^{\prime \prime}\right)\right\}
$$

Intermediate preferences We now define the intermediateness property for a family of preferences. The property is defined relative to a betweenness relationship on the set of characteristics: it requires the betweenness relationship to carry over to orders. Formally

Definition 1 Let $\Theta$ be endowed with a betweenness relationship described by the graph $G=(\Theta, E)$. The family $\mathcal{O}=\left\{R_{\theta}, \theta \in \Theta\right\}$ satisfies the intermediateness property if for any $\theta$ and $\theta^{\prime \prime}$ in $\Theta, R_{\theta^{\prime}}$ is between $R_{\theta}$ and $R_{\theta^{\prime \prime}}$ whenever $\theta^{\prime}$ is between $\theta$ and $\theta^{\prime \prime}$.

In some problems, the parameters and the graph structure are 'natural'. In the problem of locating public facilities for example, individuals' preferences are parameterized by their location on a transportation network (see for example Hansen and Thisse 1981 and the references therein). In some other problems, an appropriate parameterization exists if the family satisfies some conditions. For example, some conditions on preferences on triples guarantee that a family can be parameterized by a tree (Demange 1982). ${ }^{2}$

The set $\Theta$ is assumed to be finite to simplify the presentation. The analysis readily extends to an infinite set $\Theta$ in some situations. In the problem of locating public facilities for example, any characteristic (location) along the transportation network may be feasible making the set $\Theta$ infinite: each point on the arc defined by an edge

2 The conditions are: For every triple of alternatives, there is one which is never ranked worst by any order. 




Fig. 1 Median graphs: tree, with four-cycles

may be a feasible parameter. For a thorough analysis on this type of construction and the implication on the existence of Condorcet winners, we refer the reader to Bandelt (1985).

\subsection{Median graph, median space}

Definition 2 A median graph $G=(\Theta, E)$ is such that for every triple $(\alpha, \beta, \gamma)$ made of elements in $\Theta$, a unique vertex $\mu$ is between $(\alpha, \beta),(\beta, \gamma)$ and $(\alpha, \gamma):\{\mu\}=$ $[\alpha, \beta] \cap[\beta, \gamma] \cap[\alpha, \gamma]$ or, equivalently

$$
\begin{aligned}
d(\alpha, \beta) & =d(\alpha, \mu)+d(\mu, \beta), d(\beta, \gamma)=d(\beta, \mu)+d(\mu, \gamma) \text { and } d(\gamma, \alpha) \\
& =d(\gamma, \mu)+d(\mu, \alpha) .
\end{aligned}
$$

This vertex is the unique metric median of the triple, that is the unique point that minimizes the sum of the distance to the three elements in the triple.

The set $\Theta$ (endowed with the median graph structure $G$ ) is called median space.

Note that the median of a triple is not required to be an element of the triple, as illustrated in Fig. 1 by the triples $(\alpha, \beta, \gamma)$. Trees are median graphs. Line, a particular case of tree, is also a median graph for which the median of the triple is always a member of the triple. A median graph may admit cycles, however only of length four. Figures 1 and 2 represent various median graphs. More details are given in Sect. 2.3.

Local Condorcet characteristic/median Given a graph $G=(\Theta, E)$ on $\Theta$, the (opposite of) the distance to a point defines preferences over $\Theta$. They will be called distance-preferences or $d$-preferences. Let us consider the majority rule in the set of characteristics for the $d$-preferences. Given a $n$-characteristics profile $\left(\theta^{1}, \ldots, \theta^{n}\right)$ and two vertices $\theta, \theta^{\prime}$, let $n_{d}\left(\theta, \theta^{\prime}\right)$ be the number of characteristics in the profile that are closer to $\theta$ than to $\theta^{\prime}: n_{d}\left(\theta, \theta^{\prime}\right)=\left|\left\{i, d\left(\theta^{i}, \theta^{\prime}\right)>d\left(\theta^{i}, \theta\right)\right\}\right|$. A Condorcet characteristic is a Condorcet winner for this majority relation: $\theta^{m a j}$ is a Condorcet characteristic for the $n$-characteristics profile $\left(\theta^{1}, \ldots, \theta^{n}\right)$ if

$$
n_{d}\left(\theta, \theta^{m a j}\right) \leq n_{d}\left(\theta^{m a j}, \theta\right) \text { each } \theta \text { distinct from } \theta^{m a j} .
$$



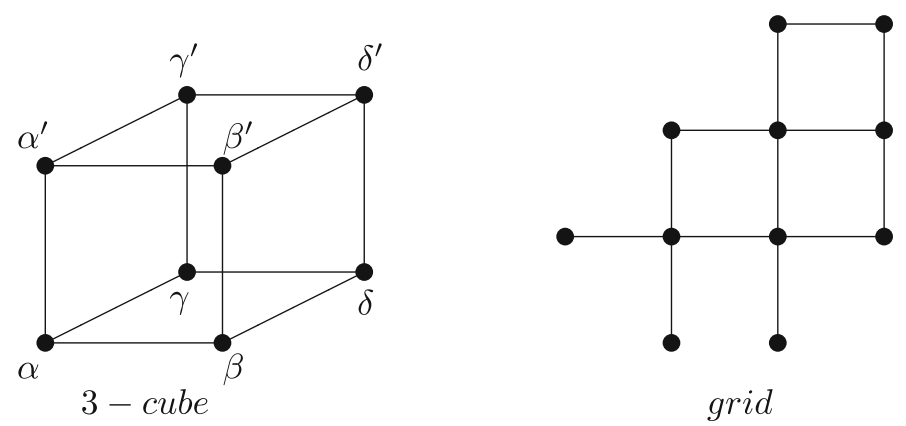

Fig. 2 Median graphs: cube, grid

A local Condorcet characteristic is only compared with its neighbors. A characteristic $\theta^{l o c}$ is a local Condorcet characteristic for the $n$-characteristics profile $\left(\theta^{1}, \ldots, \theta^{n}\right)$ if

$$
n_{d}\left(\theta, \theta^{l o c}\right) \leq n_{d}\left(\theta^{l o c}, \theta\right) \text { each } \theta \text { with }\left(\theta, \theta^{l o c}\right) \in E
$$

that is $\theta^{l o c}$ is not beaten by any of its neighbors in the graph $G$.

A key property for our analysis is that a median graph guarantees the existence of a local Condorcet characteristic, namely $\theta^{l o c}$ exists for each characteristics' profile (Bandelt and Barthélémy 1984). The local Condorcet characteristic is unique and coincides with the 'metric' median. ${ }^{3}$ Thus, if a Condorcet characteristic exists, local and global Condorcet characteristic coincide. In particular, on a line, the local Condorcet characteristic is the standard median.

Note finally that, in a median graph, a local Condorcet characteristic satisfies the stronger property that $n_{d}\left(\theta, \theta^{l o c}\right) \leq n / 2$ for any neighbor $\theta$ : no $\theta$ is at an equal distance to two adjacent points, because otherwise the graph would have an odd cycle.

\subsection{Examples}

Trees Trees are median graphs. A Condorcet characteristic exists for any profile, as can been proved in different ways (it follows from Demange 1982 since distance-preferences are single-peaked or from Bandelt and Barthélémy 1984).

Let us give a simple example of intermediate preferences family. An alternative is a pair $(\alpha, p)$ where $\alpha$ is in $\Theta$ and $p$ is a scalar. $\alpha$ can be interpreted as the 'location' for a public good and $p$ as its access price. Preferences over alternatives are represented by the utility functions

$$
u(\alpha, p, \theta)=v-p-d(\alpha, \theta) .
$$

\footnotetext{
3 The 'metric' median is a point that minimizes the sum of the distance to all characteristics in the profile. It is unique in a median graph for an odd $n$.
} 
Consider two alternatives $(\alpha, p)$ and $(\beta, q)$. We have

$$
u(\alpha, p, \theta)>u(\beta, q, \theta) \Leftrightarrow d(\beta, \theta)-d(\alpha, \theta)>p-q .
$$

Define $\Delta(\theta)=d(\beta, \theta)-d(\alpha, \theta)$. To prove that preferences are intermediate it suffices to show that for any $\theta^{\prime}$ between $\theta$ and $\theta^{\prime \prime}$ the following inequality holds

$$
\Delta\left(\theta^{\prime}\right) \geq \min \left(\Delta(\theta), \Delta\left(\theta^{\prime \prime}\right)\right)
$$

Because $\Theta$ is a tree, the interval $[\alpha, \beta]$ is the unique path joining $\alpha$ to $\beta$. Consider first the case where the three points $\theta, \theta^{\prime}$, and $\theta^{\prime \prime}$ are on this path. For any $\gamma$ on $[\alpha, \beta]$ we have $d(\alpha, \gamma)+d(\gamma, \beta)=d(\alpha, \beta)$. Hence $\Delta(\gamma)=d(\alpha, \beta)-2 d(\alpha, \gamma)$. Apply this to $\gamma=\theta, \theta^{\prime}, \theta^{\prime \prime}$. The inequality $\Delta\left(\theta^{\prime}\right) \geq \min \left(\Delta(\theta), \Delta\left(\theta^{\prime \prime}\right)\right)$ is equivalent to $d\left(\alpha, \theta^{\prime}\right) \leq \max \left(d(\alpha, \theta), d\left(\alpha, \theta^{\prime \prime}\right)\right)$. This inequality surely holds: if $\theta^{\prime}$ is not on the path between $\alpha$ and $\theta$ then $\theta^{\prime}$ is on the path between $\alpha, \theta^{\prime \prime}$. The general case follows by considering the projections of the characteristics on $[\alpha, \beta]$. Let $\widehat{\gamma}$ this projection, (which is simply here given by $m(\alpha, \gamma, \beta)$.) We have $d(\alpha, \theta)=d(\alpha, \widehat{\tau})+d(\widehat{\tau}, \tau)$ and $d(\beta, \theta)=d(\beta, \widehat{\tau})+d(\widehat{\tau}, \tau)$. hence $\Delta(\tau)=\Delta(\widehat{\tau})$. It suffices then to show that $\widehat{\theta}^{\prime}$ is between $\widehat{\theta}$ and $\widehat{\theta}^{\prime \prime}$.

Taking a fixed null price, alternatives are locations and preferences coincide with $d$-preferences. This proves that the family of $d$-preferences on a tree satisfy intermediateness.

Hypercubes An important example of median graph is a hypercube, also called a $p$-cube, which describes points with coordinates equal to 0 or 1 : the $p$-cube is $\{0,1\}^{p}$. Figure 2 represents the hypercube with 8 vertices $\{0,1\}^{3}$. Individuals characteristics are represented by an element in an hypercube in the following situation: Various dimensions affect individuals' preferences and for each one, the characteristic is binary; a dimension for instance is home ownership, marital status, worker status and so on.

The edges of the graph link the vertices the coordinates of which differ in a single value. The betweenness relationship follows: A vertex $\theta^{\prime}$ is between $\theta$ and $\theta^{\prime \prime}$ if a coordinate of $\theta^{\prime}$ is equal to 1 (respectively to 0 ) when both corresponding coordinates of $\theta$ and $\theta^{\prime \prime}$ are equal to 1 (respectively to 0 ). The median of three vertices $(\alpha, \beta, \gamma)$ is the unique point for which each coordinate equals to the majority position: coordinate $k$ of the median is equal to 1 if at least two values in the triple $\alpha_{k}, \beta_{k}, \gamma_{k}$ assume value 1 , and is equal to 0 otherwise. The median of a profile of vertices is obtained by taking the point for which each coordinate is the median of the coordinates. For example, the median of the triple $\alpha^{\prime}=(0,0,1), \delta^{\prime}=(1,1,1), \beta=(0,1,0)$ is $\gamma^{\prime}=(0,1,1)$.

Note that a Condorcet characteristic may not exist, as illustrated in Fig. 2 by the profile $\left(\alpha^{\prime}, \beta, \beta, \delta, \gamma\right)$. Characteristic $\beta$ is the local Condorcet characteristic, but is beaten by $\gamma^{\prime}$. As shown by Bandelt and Barthélémy (1984), the median graphs that guarantee a Condorcet characteristic are precisely those which are cube-free. (The graph in Fig. 3 for example is cube-free and a Condorcet characteristic always exists. For the profile with an individual on each vertex for instance, the Condorcet characteristic is $\theta^{\prime}$.) 
Fig. $3 d$-preferences are not intermediate

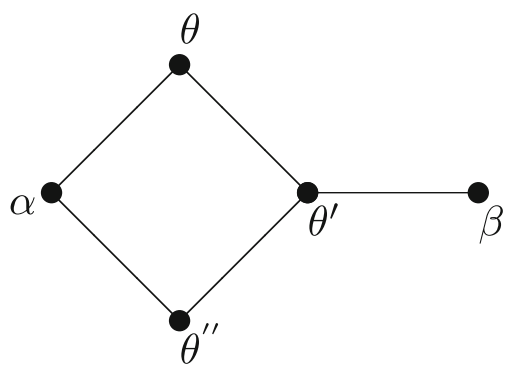

$d$-preferences A natural question is whether the $d$-preferences satisfy intermediateness. This is true if $\Theta$ is a tree, as follows from the first example in Sect. 2.3. This is not true otherwise (assuming at least five characteristics). If $\Theta$ is a median graph but not a tree, it contains a cycle of length four. With 5 points (or more), $d$-preferences are not intermediate, as illustrated in Fig. 3. Both $\theta$ and $\theta^{\prime \prime}$ prefer $\alpha$ to $\beta$ but $\theta^{\prime}$, which is between $\theta$ and $\theta^{\prime \prime}$, prefers $\beta$ to $\alpha$.

\subsection{Discrete convexity and cutting edges}

Assuming both intermediate preferences and a median parameter space has some implications. Intermediateness can be stated in terms of the discrete convexity associated with the graph. A set is said to be convex if it includes all intervals between any of two elements. With intermediate preferences, the set of characteristics for which alternative $x$ is preferred to alternative $y$ is convex.

To simplify the presentation, let us assume strict preferences. The set of characteristics are partitioned into two convex sets: those for which alternative $x$ is preferred to alternative $y$ and those for which alternative $y$ is preferred to alternative $x$. These properties are similar to those of a 'half-space' in a multi-dimensional space. Important examples of such sets are described by an edge. Given an edge $(\alpha, \beta)$ and a characteristic $\theta$, the unique median of the triple $(\theta, \alpha, \beta)$ can only be $\alpha$ or $\beta$. This is because the edge $(\alpha, \beta)$ is the unique shortest path between $\alpha$ and $\beta$ hence, by definition, the median of the triple belongs to it. Thus the set of characteristics is partitioned into two convex sets: those closer to $\alpha$ than $\beta$ and those closer to $\beta$ than $\alpha$.

We show here that intermediate preferences can be described by such 'cutting edges'. Given a graph on the parameters' space, a cutting edge represents a switch in preferences. A cutting edge for the two distinct alternatives $x$ and $y$ is an edge $(\alpha, \beta)$ such that $x$ is preferred to $y$ by the $\alpha$-ordering and $y$ is preferred to $x$ by the $\beta$-ordering. Such an edge surely exists if $x$ is not unanimously preferred to $y$ or the other way around. Intermediate preferences on a median graph are characterized by cutting edges associated to each pair of alternatives.

Lemma Let $\mathcal{O}=\left\{R_{\theta}, \theta \in \Theta\right\}$ be a family of strict preferences where $\Theta$ is a median space. A cutting edge for two alternatives $x$ and $y$ is an edge $(\alpha, \beta)$ such that $x P_{\alpha} y$ and $y P_{\beta} x$. If the intermediateness property is satisfied, then the cutting edge is unique 
and furthermore it determines the orderings over the two alternatives: $x P_{\theta} y$ if $\alpha$ is between $\theta$ and $\beta$ and $y P_{\theta} x$ otherwise.

Proof Consider a cutting edge $(\alpha, \beta)$ for the two alternatives $x$ and $y$. For every characteristic $\theta$, the unique median of the triple $(\theta, \alpha, \beta)$ is either $\alpha$ or $\beta$. If it is $\alpha$, then $\alpha$ is between $\theta$ and $\beta$ and we prove that $x P_{\theta} y$. If we had $y P_{\theta} x$, intermediate preferences and the assumption $y P_{\beta} x$ would imply $y P_{\alpha} x$, a contradiction. Hence $x P_{\theta} y$ (because preferences are strict). Similarly $y P_{\theta} x$ in the other case where $\beta$ is the median of the triple $(\theta, \alpha, \beta)$. This implies that the cutting edge is unique and characterizes the preferences over $x$ and $y$.

Let us illustrate in an hypercube. In a 3-cube as on Fig. 2, the points which are closer to $\alpha$ than to $\beta$ are those in the vertical face $\left\{\alpha, \alpha^{\prime}, \gamma, \gamma^{\prime}\right\}$. More generally, in a cube, two points linked by an edge differ in a single component, and the points which are closer to $\alpha$ than to $\beta$ are those which agree with $\alpha$ on that component. Let $\alpha$ and $\beta$ differ on the $k$-component, say with $\alpha_{k}=0, \beta_{k}=1$. If $(\alpha, \beta)$ is a cutting edge for $x$ and $y$ and preferences are intermediate, then all points whose $k$-component is null prefer $x$ to $y$ and those whose $k$-component is 1 prefer $y$ to $x$.

Projection on convex sets in a median graph Let $C$ be convex. Given $\theta$ let $\widehat{\theta}$ be a point in $C$ that minimizes the distance between $\theta$ and a point in $C$. For a median graph, $\widehat{\theta}$ has nice properties and can be qualified as the projection of $\theta$ on $C$ (it is often called a 'gate' in graph theory): It is unique and furthermore ${ }^{4}$ it is between $\theta$ and every element in $C$ :

$$
d(\theta, \gamma)=d(\theta, \widehat{\theta})+d(\widehat{\theta}, \gamma) \text { every } \gamma \in C
$$

\section{Median representative rule}

By combining a median graph structure for the set of characteristics and the property of intermediate preferences, one obtains the following theorem.

Theorem 1 Let $\Theta$ be a median space and let $\mathcal{O}=\left\{R_{\theta}, \theta \in \Theta\right\}$ be a family of intermediate preferences on a set of alternatives $X$. Let $n$ be odd and $\theta^{\text {loc }}$ be the local Condorcet characteristic of the profile $\left(\theta^{1}, \ldots, \theta^{n}\right)$.

(a) If preferences are strict, then the majority relation $P^{m a j}$ coincides with $P_{\theta}$ loc:

$$
x P^{m a j} y \text { if and only if } x P_{\theta} \text { loc } y \text {. }
$$

\footnotetext{
4 The proof that $\widehat{\theta}$ is unique is easy. By contradiction let $\theta_{1}$ and $\theta_{2}$ be two distinct points in $C$ whose distance to $\theta$ is minimum. Consider the median $\mu$ of the three points $\left(\theta, \theta_{1}, \theta_{2}\right)$. Since $\mu$ is between $\theta_{1}$ and $\theta_{2}, \mu$ is in $C$ by convexity. Applying the conditions (4) to the triple $\left(\theta, \theta_{1}, \theta_{2}\right)$ we also have $d\left(\theta, \theta_{1}\right)=d(\theta, \mu)+d\left(\mu, \theta_{1}\right)$ and $d\left(\theta, \theta_{2}\right)=d(\theta, \mu)+d\left(\mu, \theta_{2}\right)$. By definition, since $\mu$ is in $C, d\left(\theta, \theta_{1}\right) \leq d\left(\theta_{1}, \mu\right)$ so we must have $d\left(\mu, \theta_{1}\right)=0$, i.e. $\mu$ coincides with $\theta_{1}$ and similarly for $\theta_{2}$, this gives the contradiction.

Given $\gamma$ an element of $C$, a similar argument implies that $\widehat{\theta}$ must be the median of the triple $(\theta, \widehat{\theta}, \gamma)$ : this proves that $\widehat{\theta}$ is between $\theta$ and $\gamma$, hence (7).
} 
(b) If $\Theta$ is a tree (and preferences not necessarily strict)

$$
x P^{m a j} y \text { implies } x R_{\theta} \text { loc } y \text {. }
$$

When preference orderings are strict, the majority relation is strict as well (because $n$ is odd) and exactly coincides with the order associated with the characteristic $\theta^{l o c}$ : $\theta^{l o c}$ can be qualified as a representative characteristic.

When preference orderings are not strict, the majority relation and the ordering associated with $\theta^{l o c}$ never rank a pair in opposite order. This implies that if the preference $R_{\theta} l o c$ has a unique top alternative, this alternative is a Condorcet winner. The two orderings however may not coincide, as illustrated by the following example. There are three characteristics on a line $\alpha, \beta, \gamma$ with $\beta$ between $\alpha$ and $\gamma$. Let two alternatives $x$ and $y$ for which $x P_{\alpha} y, y I_{\beta} x$, and $y P_{\gamma} x$ where $I$ denotes indifference. Take five individuals, 2 at $\alpha, 2$ at $\beta$, and 1 at $\gamma$ so $\beta$ is the winner characteristic, here the usual median. Then $x$ is preferred to $y$ by the majority but not by the median.

Theorem 1 fails when the condition (b) is not required on intermediate preferences (note that this condition only matters for preferences that are not strict orderings). The following simple example illustrates this point. Take as above three characteristics on a line $\alpha, \beta, \gamma$ and two alternatives $x$ and $y$. Let $x P_{\alpha} y, y P_{\beta} x$, and $x I_{\gamma} y$. Condition (a) is met but (b) is violated. Take five individuals, 2 at each extreme $\alpha$ or $\gamma$ and 1 at the winner characteristic $\beta$. Then $x$ is preferred to $y$ under the majority relation but the median prefers $y$ to $x$.

The characteristic $\theta^{l o c}$ is independent of the voting problem at hand, provided that the same parameter space commands the preferences. A similar property holds true when preferences are single-peaked on a line: the median characteristic is independent of the voting problem so that its peak is a Condorcet winner (but the median may not be a representative characteristic simply because preferences are not characterized by their peak).

Finally, the transitivity of the majority relation when the characteristics are on a tree can also be derived from a more general result on the core. The core of any superadditive game built on intermediate preferences on a tree is non-empty (Demange 1994). In particular the core of a majority game is non-empty under intermediate preferences. Since the core of a majority game can only contain a Condorcet winner, this implies the existence of a Condorcet winner. An application of the same argument to any subset of alternatives proves the transitivity of the majority rule.

Proof The proof is by contradiction. Let $x$ beat $y$ for the majority rule and assume that $y$ is (weakly) preferred to $x$ by $\theta^{l o c}: x P^{m a j} y$ and $y R_{\theta} l o c x$. Denote $n=2 k+1$.

We first show that $y R_{\theta} l o c x$ implies that the number of individuals who strictly prefer $x$ to $y$ is smaller than $n / 2$ : $\left|\left\{i, x P_{\theta^{i}} y\right\}\right| \leq k$ or denoting by $C$ the set of characteristics for which $x$ is strictly preferred to $y$ :

$$
\left|\left\{i, \theta_{i} \in C\right\}\right| \leq k \text { where } C=\left\{\theta, x P_{\theta} y\right\}
$$

The set $C$ is convex and by assumption $\theta^{l o c}$ does not belong to $C$. Consider the projection $\widehat{\theta}^{l o c}$ of $\theta^{l o c}$ on $C$. There is $\mu$ neighbor of $\theta^{l o c}$ on $\left[\widehat{\theta}^{l o c}, \theta^{l o c}\right]$, i.e. on a shortest 

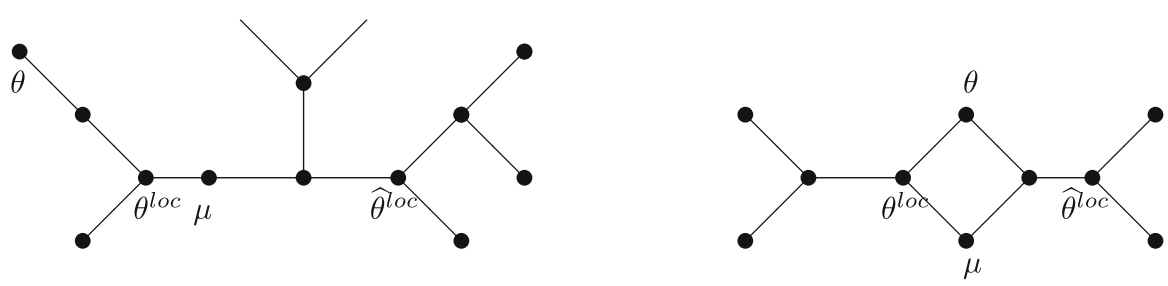

Fig. $4 \theta$ closer to $\theta^{l o c}$ than to $\mu$ : $\theta^{l o c}$ is between $\theta$ and $\widehat{\theta}^{l o c}$ (left) or not (right)

path between $\widehat{\theta}^{l o c}$ and $\theta^{l o c}$, hence $d\left(\mu, \theta^{l o c}\right)=d\left(\mu, \widehat{\theta}^{l o c}\right)+1$. We show that $\mu$ is closer to each element $\gamma$ in $C$ than $\theta^{l o c}$. Standard distance inequality $d(\mu, \gamma) \leq$ $d\left(\mu, \widehat{\theta}^{l o c}\right)+d\left(\widehat{\theta}^{l o c}, \gamma\right)$ applies, hence

$$
d(\mu, \gamma) \leq d\left(\theta, \widehat{\theta}^{l o c}\right)-1+d\left(\widehat{\theta}^{l o c}, \gamma\right)
$$

Now, since $\widehat{\theta}^{l o c}$ is the projection of $\theta^{l o c}$ on $C$, (7) holds for $\gamma \in C: d\left(\theta^{l o c}, \gamma\right)=$ $d\left(\theta^{l o c}, \widehat{\theta}^{l o c}\right)+d\left(\widehat{\theta}^{l o c}, \gamma\right)$, and we obtain

$$
d(\mu, \gamma) \leq d\left(\theta^{l o c}, \gamma\right)-1 \text { every } \gamma \in C \text {. }
$$

Thus the characteristics in $C$ are closer to $\mu$ than to $\theta^{l o c}$. By assumption $\theta^{l o c}$ is a local Condorcet characteristic, hence it is not beaten by its neighbor $\mu$ for the distancepreferences: the number of characteristics in the profile closer to $\mu$ than to $\theta^{l o c}$ is not greater than $n / 2$, i.e. than $k$. This implies $\left|\left\{i, \theta_{i} \in C\right\}\right| \leq k$, which proves (10), the desired result.

(a) follows: If preferences are strict, $x$ beats $y$ by a majority implies that the number of individuals who strictly prefer $x$ to $y$ is strictly larger than $k:\left|\left\{i, \theta_{i} \in C\right\}\right|>k$, in contradiction with (10). This proves that $x P^{m a j} y$ implies $x P_{\theta}$ loc $y$. Since both relations $P^{m a j}$ and $P_{\theta}$ loc are strict, they coincide.

For (b) we assume $\Theta$ is a tree and furthermore that $y P_{\theta l o c} x$ to get the contradiction. Take a characteristic $\theta$ distinct from $\theta^{l o c}$ that is closer to $\theta^{l o c}$ than to $\mu$. We show that $\theta^{l o c}$ is between $\theta$ and $\widehat{\theta}^{l o c}$ as illustrated in Fig. 4 on the left (the property fails in general median spaces as illustrated in the graph on the right) and that $y P_{\theta} x$. The path from $\theta^{l o c}$ to $\widehat{\theta}^{l o c}$ is: $\theta^{l o c}, \mu, \ldots, \widehat{\theta}^{l o c}$. The path from $\theta$ to $\theta^{l o c}$ does not contain $\mu$, (because $\theta$ is closer to $\theta^{l o c}$ than to $\mu$ ). Hence the two paths from $\theta$ to $\theta^{l o c}$ and from $\theta^{l o c}$ to $\widehat{\theta}^{l o c}$ intersect only at $\theta^{l o c}$ : thus the unique path joining $\theta$ to $\widehat{\theta}^{l o c}$ is formed by their union. This proves that $\theta^{l o c}$ is between $\theta$ and $\widehat{\theta}^{l o c}$. This implies that $y P_{\theta} x$ thanks to intermediate preferences, and the fact that $x P_{\widehat{\theta}}$ loc $y$ and $y P_{\theta l o c} x$.

So we have proved that $y P_{\theta} x$ for all $\theta$ closer to $\theta^{l o c}$ than to its neighbor $\mu$. Since $\theta^{l o c}$ is a local Condorcet characteristic there are at least $k+1$ such characteristics, ${ }^{5}$ at least as many as those in $C ; x P^{m a j} y$ is impossible.

Two consequences can be drawn from Theorem 1.

\footnotetext{
${ }^{5}$ We use the fact here that a point is never at the same distance of two points linked by an edge, as shown in Sect. 2.4.
} 
First, as is well known, a majority relation that is transitive and strict on a product domain of preferences is not subject to the standard difficulties of aggregation and manipulation of preferences. Specifically the majority relation satisfies all the axioms of Arrow theorem and furthermore the associated mechanism is strategy-proof. We state this last result as a Corollary and give the proof for completeness. Here the mechanism asks to each individual a characteristic (or alternatively a preference in $\mathcal{O}$ ) and selects the alternative that is preferred by the majority order of the announced profile, i.e., the top alternative for $\theta^{l o c}$.

Corollary Let $\Theta$ be a median space and let $\mathcal{O}=\left\{R_{\theta}, \theta \in \Theta\right\}$ be a family of intermediate strict preferences on a set of alternatives $X$. Let $n$ be odd and $\theta^{\text {loc }}$ be the local Condorcet characteristic of the profile $\left(\theta^{1}, \ldots, \theta^{n}\right)$. The majority mechanism, which assigns to each $\left(\theta^{1}, \ldots, \theta^{n}\right)$ the top alternative of $\theta^{\text {loc }}$, is strategy-proof.

Proof Let $\tau$ the profile obtained from $\theta=\left(\theta^{1}, \ldots, \theta^{n}\right)$ by changing $\theta^{1}$ by $\tau^{1}$, and let $y$ and $x$ the majority alternatives respectively associated to $\tau$ and $\theta$. Strategy-proofness requires that individual 1 does not benefit from announcing $\tau^{1}$ instead of $\theta^{1}$ if his true characteristic is $\theta^{1}: x R_{\theta^{1}} y$. Suppose by contradiction $y P_{\theta^{1}} x$. Then, since $x$ is a Condorcet winner at profile $\theta$, there are more individuals in $2, \ldots, n$ who prefer $x$ to $y$. But this is also true at profile $\tau$ since the characteristics of these individuals are unchanged.

Second, Theorem 1 bears on the majority relation but readily extends to some variations. Specifically, consider a relation that is obtained by adding 'phantom' voters in a similar way as in Moulin (1980). Fix a profile $\left(\tau^{1}, \ldots, \tau^{p}\right)$, the profile of $p$ phantom voters. The relation is defined as the majority relation of the extended profile with $n+p$ characteristics obtained by adding the fixed profile $\left(\tau^{1}, \ldots, \tau^{p}\right)$ to the individual characteristics' profile $\left(\theta^{1}, \ldots, \theta^{n}\right)$. Under the assumptions of Theorem 1 , the relation satisfies the same properties since it is the majority rule over $\mathcal{O}^{n+p}$ restricted to some profiles. Hence the aggregation and strategy-proof results hold for these modified majority relations.

\subsection{Concluding remarks}

It is useful to clarify the differences between single-peaked and intermediate preferences and the types of associated results. For that we use the tree example. The set of alternatives $X$ is endowed with a tree structure. To simplify consider strict preferences. A preference ordering on $X$ with top alternative $x$ is single-peaked if the ordering decreases along any path starting at $x$ : if $y$ is between $x$ and $z$ surely $y$ is preferred to $z$. A Condorcet winner always exists for single-peaked preferences on a tree (Demange 1982). The majority relation however may not be transitive as illustrated by the following example. Consider a star with four points with $t$ in the center. Observe that distinct orders may have the same top alternative, so the family is not indexed by $X$. Here the set of single-peaked orders is composed with all the orders which rank $t$ first or second. The set does not guarantee the transitivity of the majority relation as illustrated with three individuals and preferences: if $N=\{1,2,3\}, t P^{1} x P^{1}$ y $P^{1} z$, $t P^{2} y P^{2} z P^{2} x, t P^{3} z P^{3} x P^{3} y$. The majority relation has a cycle on $\{x, y, z\}$ (Fig. 5). 
Fig. 5 No restriction on the ranking of $x, y, z$ for single-peakedness preferences

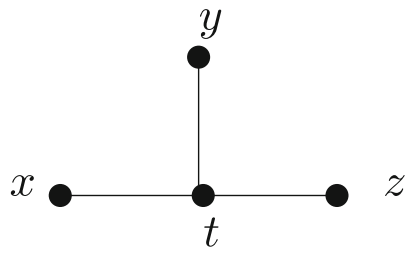

Consider now a family of intermediate preferences indexed by a point running on the tree where the index represents the peak of the preference. A single relation has $t$ as the peak. This precludes the above example with the (dominated) Condorcet triple and the non-transitivity of the majority rule. Although this example may suggest that the framework with intermediate preferences is more restrictive than the one with single-peaked preferences, this is not true since intermediate preferences allow for any set of alternatives. This fact has been somewhat blurred because the line model is the prominent model. In that case, although the distinction exists, it may be thought to be rather mild. This is definitely not the case when the sets $\Theta$ and $X$ are distinct.

To conclude, given the tremendous literature on the majority rule, there is little hope to find exciting and entirely new families that guarantee the existence of a Condorcet winner. Our results can be seen as extending some of the results that have been obtained when the characteristics are ordered on a line or on a tree to more general median graphs, in particular to hypercubes which can be a good representation of qualitative characteristics. It remains to interpret intermediateness in these contexts and to see whether there are interesting applications.

Open Access This article is distributed under the terms of the Creative Commons Attribution License which permits any use, distribution and reproduction in any medium, provided the original author(s) and source are credited.

\section{References}

Arrow KJ (1963) Social choice and individual values, 2nd edn. Yale University Press Bandelt HJ (1985) Networks with Condorcet solutions. Eur J Oper Res 20:314-326

Bandelt H-J, Barthélémy JP (1984) Medians in median graphs. Discrete Appl Math 8(2):131-142

Barbera S, Moreno B (2009) Top monotonicity: a common root for single peakedness, single crossing and the median voter result. Working paper

Black D (1948) On the rationale of group decision-making. J Political Econ 56:23-34

Demange G (1982) Single-peaked orders on a tree. Math Soc Sci 3:389-396

Demange G (1983) Spatial models of collective choice. In: Thisse JF, Holler H-G (eds) Locational analysis of public facilities. North Holland, pp 153-182

Demange G (1994) Intermediate preferences and stable coalition structures. J Math Econ 23:45-58

Grandmont J-M (1978) Intermediate preferences and the majority rule. Econometrica 46:317-330

Hansen F, Thisse JF (1981) Outcomes of voting and planning: Condorcet. Weber and Rawls locations. J Public Econ 16:1-15

Kemeny JG, Snell JL (1962) Mathematical models in the social sciences. Ginn, New York

Kramer GH (1973) On a class of equilibrium conditions for majority rule. Econometrica 41:285-297

Moulin H (1980) On strategy-proofness and single-peakedness. Public Choice 35(4):437-455

Mulder HM (1980) The interval function of a graph, vol. 132. Centrum Voor Wiskunde en Informatica, pp. 1-191. http://repository.cwi.nl/search/fullrecord.php?publnr=12991 
Nehring K, Puppe C (2007) The structure of strategy-proof social choice. Part I: general characterization and possibility results on median spaces. J Econ Theory 135:269-305

Plott CR (1967) A notion of equilibrium and its possibility under majority rule. Am Econ Rev 57:788-806 Van De Vel MLJ (1993) Theory of convex structures. North Holland 